\title{
Simon Guntrum
}

\section{Frankreichs Widersacher, Deutschlands "bad cop"? Die Formierung der "New Hanseatic League" in den jüngsten Reformdiskussionen der Eurozone}

Häufig wird in den Debatten über die Zukunft der europäischen Integration auf die zentrale Bedeutung des deutschfranzösischen "Motors“ verwiesen. Der PROKLA-Beitrag von Schneider/ Syrovatka (2017) konstatiert, bezugnehmend auf jüngste Debatten über die Reformierung der Wirtschafts- und Währungsunion (WWU), eine Blockade des deutsch-französischen Verhältnisses. Hintergrund der Reformdebatten ist eine nach wie vor - trotz Einführung des Europäische Stabilitätsmechanismus (ESM) als dauerhafte Finanzierungsinstitution und trotz Fortschritten in der Bankenunion prekäre Architektur der Eurozone. Infolge der Eurokrise traten die Krisenanfälligkeit der Währungsunion und die in ihr eingeschriebenen ökonomischen Asymmetrien zwischen den Mitgliedstaaten offen zutage. Auch wenn sich die Lage wieder etwas beruhigt hat, zeigen Entwicklungen wie der Wahlsieg Syrizas 2015 und die jüngsten Spannungen zwischen Italien und der EU, dass die WWU nach wie vor auf wackeligen Beinen steht. Um die Eurozone krisenfester zu machen, bestimmen nach Schneider/Syrovatka (2017) zwei gegensätzliche wirtschaftspolitische Leitbilder die Reformdebatte, die zugleich eine Ursache der Blockade des deutsch-französischen Verhältnisses sind: Einer maßgeblich von Frankreich geforderten „Fiskalunion“, die weitreichende Integrationsschritte erfordert, stünde eine von Deutschland präferierte "Stabilitätsunion“ gegenüber, die eine strikte Einhaltung von Fiskalregeln und eine nationale Verantwortung in der Finanzpolitik betont.

Zweifelsohne war das deutsch-französische Verhältnis in der Geschichte der EU ein zentraler Impulsgeber für Integrationsprozesse. Ein Fokus auf dieses Verhältnis verstellt allerdings den Blick auf ein erweitertes Feld zwischenstaatlicher Kräfteverhältnisse. Ein Blick über das deutsch-französische Verhältnis hinaus offenbart Koalitionsdynamiken der anderen 26 Mitgliedstaaten der EU, die die Reformdebatten mitbestimmen und die sich zusammenschließen können, um gemeinsamen Reformvorstellungen stärkeres Gehör zu verschaffen.

$\mathrm{Zu}$ diesem Zweck hat sich jüngst eine Gruppierung hervorgetan, die ehemals als the bad weather coalition (die Schlechtwetterkoalition) oder the vikings (die Wikinger) (Financial Times 2018d) bekannt wurde, aber neuerdings vermehrt als the new hanseatic league (die neue hanseatische Liga, im Folgenden Hansa) in Erscheinung tritt. Hierbei handelt es sich um einen Zusammenschluss der EU-Mitgliedstaaten Niederlande, Irland, Dänemark, Schweden, Finnland sowie der baltischen Staaten Estland, Lettland und 
Litauen. Die Namensgebung geht dabei zurück auf den Verbund von Kaufleuten und Hansestädten in der frühen Neuzeit, die ein Handelsmonopol über die Seewege der Nordsee und des baltischen Meeres etablieren konnten.

Besondere Aufmerksamkeit wurde der Hansa beim Treffen der Eurogruppe Anfang Dezember 2018 zuteil, als sie mit dem französischen Finanzminister Bruno Le Maire aneinandergeriet. Knackpunkt der Verhandlungen war die Zukunft der Eurozone und die Einführung eines gemeinsamen Eurozonenbudgets, das ganz und gar nicht den Vorstellungen der Hanseaten entsprach, und die sich dementsprechend in den Reformdiskussionen zum Gegenspieler Frankreichs entwickelten (Handelsblatt 2018b).

Der Artikel soll eine erste Bestandsaufnahme leisten und zeigen, auf welche Dynamiken die Formierung der Hansa zurückzuführen ist, welche Positionierung sie in den Reformdebatten einnimmt, und ob die Hansa lediglich den verlängerten Arm der deutschen Stabilitätsunion oder doch einen eigenständigen Widersacher der französischen Reformbestrebungen darstellt.

\section{Schiffstaufe: die Gründung der "Hansa"}

Die Gründung der Hansa lässt sich auf den 6. März 2018 datieren. In dieser Zeit nahmen die Reformdebatten über die Zukunft der Eurozone kräftig an Fahrt auf ${ }^{1}$. Frankreichs Präsident Macron machte im September 2017 mit seiner viel beachteten Rede an der Pariser Sorbonne-Universität

1 Ein sehr guter Überblick über die einzelnen Reformvorschläge findet sich bei Dullien (2018). den Aufschlag, in der er neben der Einführung einer europäischen Finanztransaktionssteuer ein eigenes Budget, einen Finanzminister bzw. eine Finanzministerin sowie ein Parlament für die Eurozone forderte (Macron 2017). Eine Reaktion seitens der deutschen Bundesregierung ließ lange auf sich warten. Erst in einem Interview in der Frankfurter Allgemeinen Sonntagszeitung vom 3. Juni 2018 reagierte die Bundeskanzlerin Angela Merkel und zeigte sich prinzipiell offen für eine Umwandlung des ESM zu einem Währungsfonds sowie für die Einführung eines Investivhaushalts für die Eurozone (FAS 2018). Daraufhin trafen sich am 19. Juni 2018 VertreterInnen der deutschen und französischen Regierung auf Schloss Meseberg, um gemeinsame Positionen in der Reformdiskussion abzustecken. Die Ergebnisse wurden im „Deutsch-Französischen Fahrplan für das Euro-Währungsgebiet“ (BMF 2018) zusammengefasst. Überraschend umfangreich enthält diese Erklärung die Einführung eines Eurozonen-Haushalts und eines Europäischen Stabilisierungsfonds für Arbeitslosigkeit sowie einen Ausbau der Kompetenzen des ESM, der zudem eine neue Kreditlinie für Länder in konjunktureller Schieflage vorsieht (Dullien 2018).

In der Zwischenzeit machte auch die Hansa erstmals auf sich aufmerksam. In einem gemeinsam von den Finanzministern der Hansastaaten veröffentlichten Dokument zur Diskussion über die Eurozonenarchitektur legte sie die Grundelemente ihrer Reformvorschläge dar (Finnish Ministry of Finance 2018). Es folgten weitere Positionspapiere zum Projekt der Kapitalmarktunion (Irish Department of Finance 2018) und zur künftigen Rolle des Europäischen Stabilitätsmechanismus (Dutch Ministry of Finance 2018). Diese Positionspapiere sind in den Kontext spezifischer Eurogipfel zu stellen, insbesondere 
den Gipfeln vom 29. Juni 2018 und vom 14. Dezember 2018, in denen dezidiert die Frage der Zukunft der Währungsunion auf der Tagesordnung stand.

\section{Der Kurs der Hansa in rauen Reformgewässern: zwischen deutscher Stabilitätsunion und britischem Freihandel}

Um die wirtschaftspolitische Positionierung der Hansa zu analysieren und um zu klären, wie sie sich hinsichtlich der deutsch-französischen Achse einordnen lässt, bedarf es zunächst einer knappen Darlegung der zwischenstaatlichen Blockbildung in der EU.

Wenn man Schneider/Syrovatka (2017) und Heine/Sablowski (2015) folgt und die ungleiche Entwicklung in der Eurozone als strukturelle Determinante für eine Bildung von Staatenblöcken zugrunde legt, lassen sich prinzipiell zwei Blöcke in der Eurozone identifizieren. Zum einen den auf den Binnenmarkt ausgerichteten südeuropäischen Block, der sich um Frankreich bildet. Aus den Ökonomien dieses Blocks kommen meistens Fürsprecher einer Fiskalunion mit weiteren Kompetenzübertragungen auf die europäische Ebene, insbesondere durch die Einführung eines Eurozonenhaushalts und anderer fiskalischer Mechanismen der Risikoteilung. Demgegenüber steht der Block aus nordeuropäischen Staaten, die sich um Deutschland formieren und eng in seine exportorientierten Produktionsstrukturen eingebunden sind, wie etwa auch die Visegrádgruppe aus der Slowakei und den Nicht-Eurostaaten Polen, Ungarn und Tschechien (Becker 2018). Der besagte "deutsche Block“ ist jedenfalls in Fragen der Reform der Währungsunion weniger auf einen großen Wurf aus als auf das strikte Einhalten haushaltspolitischer Vorgaben, die Durchführung einer restriktiven Haushaltspolitik und die Ablehnung jeglicher fiskalischer Risikoteilung auf europäischer Ebene (Bieling 2011: 185f.).

Weitet man den Blick über die Eurozone hinaus, dürfte Großbritannien einen dritten Block in dieser Konstellation darstellen. Spätestens mit der konservativen Wende der Thatcherregierung in den 1980er Jahren trat Großbritannien für eine umfassende Liberalisierungsagenda ein, insbesondere durch Deregulierungen und Marktöffnungen in den Bereichen der Finanzmärkte und -dienstleistungen (Helleiner 1994). Obwohl Großbritannien tendenziell eher dem deutschen Block zugewandt war, kann es diesem nicht klar zugeordnet werden, da es stets Vorbehalte gegenüber einer vertiefenden Integration hin zu einer „ever closer union“, einer immer engeren Bindung, äußerte, den Beitritt zur Währungsunion durch eine ,opt-outKlausel“ ablehnte und nicht selten Sonderrechte einforderte (Macchiarelli 2017).

In dieser Konstellation verorteten sich die acht Hansastaaten, je nach Politikfeld, zwischen Deutschland und Großbritannien. Zwei Entwicklungen sind dafür ursächlich, dass sich jüngst eine eigenständige hanseatic league herausbilden konnte. Einerseits stand die Hansa in Fragen der Handelsliberalisierung stets auf der Seite Großbritanniens. Wie der niederländische Finanzminister Wopke Hoekstra in einem Interview klarstellte, haben die Aktivitäten der Hansa daher auch mit dem Austritt Großbritanniens aus der EU zu tun (Handelsblatt 2018a). Die Niederlande - als eine der Initiatorinnen der Hansa konnten sich in der Vergangenheit stets auf die starke Position des europäischen Schwergewichts Großbritannien in Freihandelsfragen verlassen. Durch den Brexit geht aber ein zentraler Fürsprecher freier Märkte verloren, weshalb die Niederlande 
nun mit anderen, vor allem den nordeuropäischen und baltischen Staaten, neue Allianzen schmieden müssen, um ihren Forderungen weiter Nachdruck zu verleihen. Die acht Hanseaten teilen diesen „Einsatz für den Freihandel“ (Hoekstra zit. n. ebd.) der beispielsweise in einer gemeinsamen Positionierung zur Kapitalmarktunion deutlich wird. Dieses Projekt zur Integration der Kapitalmärkte war ein zentrales Anliegen Großbritanniens, aber auch Irlands, Schwedens und der Niederlande (Quaglia u.a. 2016). Aufgrund des Brexits tritt die Hansa nun in Großbritanniens Fußstapfen und treibt die Kapitalmarktunion selbständig voran (Irish Department of Finance 2018).

In Fragen der wirtschaftspolitischen Reformen und der Zukunft der Eurozonenarchitektur vertritt die Hansa mit Deutschland vergleichbare Standpunkte. Im Schatten der politischen und ökonomischen Durchschlagskraft Deutschlands fühlen sich die Hanseaten relativ wohl, wie auch die Niederlande stets betonen: „Deutschland und die Niederlande haben bei fast allen finanzpolitischen Themen die gleiche Position." (Hoekstra zit. n. Handelsblatt 2018a). Wirtschaftspolitisch stehen die Hanseaten dementsprechend auch für zentrale von Deutschland propagierte Paradigmen: nationale Verantwortung für öffentliche Finanzen, ein starker Fokus auf fiskalische Regeleinhaltung, insbesondere im Rahmen des Stabilitäts- und Wachstumspakts, sowie eine generelle Skepsis gegenüber fiskalischer Risikoteilung (Financial Times 2018d; The Economist 2018).

Konkreter zeigt sich diese wirtschaftspolitische Ausrichtung in den bisher von der Hanseatischen Liga veröffentlichten Positionspapieren, insbesondere in jenen zur Zukunft der Währungsunion und zur Reform des ESM. Darin wird umfangreichen Reformen der Eurozone, wie sie Frankreich fordert, eine deutliche Absage erteilt. Die Mitgliedstaaten werden vielmehr dazu aufgefordert, sich auf die konsequente Umsetzung bestehender Reformen zu konzentrieren, wie etwa einer Vollendung der Bankenunion, einer weiteren Vertiefung des Binnenmarkts durch die Kapitalmarktunion sowie einer Transformation des ESM hin zu einem Europäischen Währungsfonds (Dutch Ministry of Finance 2018; Finnish Ministry of Finance 2018; Irish Department of Finance 2018). Diese schrittweisen Reformen „sollten Vorrang vor weitreichenden Vorschlägen haben "(Finnish Ministry of Finance 2018: 1, Übers.: S.G.).

Offensichtlich besteht in diesen Punkten große Übereinstimmung mit Deutschland. Das zeigt sich über den ESM hinaus auch in Bezug auf die Einführung einer gemeinsamen europäischen Einlagensicherung (EDIS) als letzten Pfeiler der Bankenunion (vgl. Howarth/Quaglia 2017). Die Hansa, wie auch Deutschland, sperrt sich bisher gegen Fortschritte, auch wenn sie prinzipiell einem Fahrplan und der Ausarbeitung weiterer technischer Details zustimmt. Letztlich ist auch hier die Position persistent, dass nationale Bankenrisiken, insbesondere in der Form fauler Kredite, zunächst abgebaut werden müssen, ehe man einer gemeinsamen Einlagensicherung zustimmen könne. Ab welchem Schwellenwert einer Risikoreduktion Deutschland und die Hansa einer EDIS zustimmen würden, bleibt allerdings unklar (Vestergaard 2018).

Darüber hinaus spricht sich die Hansa für eine Ausweitung der Kompetenzen des ESM aus. Wo bisher die Kommission die Hoheit über die Bewertung der Schuldentragfähigkeit von Ländern innehat, müsse der ESM die Rolle der Kommission zusätzlich ergänzen. Die Hansa fordert 
eine stärkere Rolle des ESM bei der Überwachung und Kontrolle der nationalen Fiskalpolitik - eine Position, die allerdings auch Deutschland und Frankreich in der Meseberger Erklärung äußerten (BMF 2018: 1) Unterschiede gibt es jedoch bei den Konditionalitäten, an die die Gewährung von Finanzhilfen an einen finanziell notleidenden Staat gekoppelt sind. Nach Vorstellung der Hansa solle jeder Gewährung von Finanzhilfe über den ESM eine - durch den ESM selbst durchgeführte - Analyse der Risiken für die Rückzahlungsfähigkeit des jeweiligen Staates, der Hilfe beansprucht, vorausgehen und der Staat eine Schuldenrestrukturierung durchführen, ehe er eine Finanzhilfe in Anspruch nehmen könne (Dutch Ministry of Finance 2018; Financial Times 2018c). In diesem Aspekt unterscheidet sich die Hansa dann auch von Frankreich, das eine solche Restrukturierung vor der Gewährung von Finanzhilfen als ,inakzepabel" ablehnt (Financial Times 2018c, Übers.: S. G.)

Die gemeinsamen Statements der acht Hanseaten offenbaren jedoch auch Abgrenzungen gegenüber Deutschlands Positionierung. Beispielsweise lehnt die Hansa die Einführung eines Eurozonenbudgets weitestgehend $\mathrm{ab}$ und ist hier zu wenig $\mathrm{Zu}$ geständnissen gegenüber Frankreich bereit (Financial Times 2018a, 2018b). Ähnliches lässt sich im Bereich der Dienstleistungen beobachten. Die Hansa fordert eine stärkere Öffnung der nationalen Dienstleistungssektoren, um den Binnenmarkt zu vollenden, was Frankreich und Deutschland bislang blockieren (Financial Times 2018d).

Was die Ergebnisse der Gipfel anbelangt, kann man der Hansa gewisse Teilerfolge bescheinigen. Einigungen gab es lediglich bezüglich einer Letztsicherung für den Abwicklungsfonds insolventer
Banken und einer Kompetenzausweitung des ESM. Ihr größter Erfolg ist sicherlich, dass der von Frankreich geforderte große Wurf an Maßnahmen hin zu einer umfangreichen Fiskalunion verhindert oder zumindest abgeschwächt werden konnte. Einen europäischen Finanzminister oder eine Finanzministerin wird es ebenso weniggeben wie ein Eurozonenparlament, das über ein umfassendes Eurozonenbudget verfügen kann. Letzteres konnte die Hansa zwar nicht ganz verhindern. Das zukünftige Haushaltsinstrument ist zunächst, wie die europäische Einlagensicherung, auf die lange Bank verschoben worden. Zudem soll es im niedrigen zweistelligen Milliardenbereich verbleiben, weshalb es mehr ein „Budgetchen“ als ein Zugeständnis an Macrons Forderung nach einem substantiellen Haushalt für die Eurozone ist (Europäischer Rat 2018)

Auch wurde die Rolle des ESM im Gipfelbeschluss vom 14. Dezember 2018 reformiert. Zukünftig erhält der ESM zusätzliche Aufsichts- und Kontrollaufgaben für Staaten, die sich einem Anpassungsprogramm unterziehen (müssen). Dabei soll der ESM neben der EU-Kommission fortan die Konzeptionierung, Verhandlung und Kontrolle der Programme übernehmen, die Schuldentragfähigkeit mitbewerten und letztlich die "Troika" weitestgehend ersetzen (ESM 2018), was auch eine zentrale Forderung der Hansa war.

\section{Die Hansa als ein "bad cop" Deutschlands? Implikationen für zukünftige Euro-Reformen}

Sicherlich ist der Zusammenschluss der acht Staaten zur hanseatic league ein Versuch, den Interessen dieser Staaten stärkeres Gewicht in der sonst sehr durch Frankreich und Deutschland geprägten Eurodebatte zu verschaffen. Dahingehend 
und auch aufGrund partieller Abweichungen gegenüber der deutschen Positionierung könnte man zu dem Urteil kommen, die Hansa handle gegen den deutsch-französischen Motor.

Naheliegender ist es jedoch, bei der Hansa von einer unterstützenden Kraft der deutschen Position zu sprechen. Man gewinnt den Eindruck, den Verhandlungsergebnissen der Eurogipfel liege das Spiel des good cop versus bad cop zugrunde, wobei sich Deutschland die erste und der Hansa die zweite Rolle zuweisen ließe. Klar ist: Die deutsche Position hat sich weitestgehend durchgesetzt. Macrons Visionen einer umfangreichen Reform der Währungsunion sind größtenteils gescheitert. Weitreichende, progressive Reformen, die einen fiskalischen Risikoausgleich zwischen den Euroländern vorsehen und strukturelle Divergenzen zwischen den Mitgliedstaaten reduzieren, bleiben aus (vgl. Bieling/Guntrum 2019). Aus einer Perspektive, die auf einen solidarischen Lastenausgleich und eine europäisch koordinierte Wirtschafts- und Sozialpolitik setzt, ist das Spiel auch eher als eines zwischen bad cop (Deutschland) und worse cop (Hansa) zu interpretieren. Wenn man den Gegensatz von Schneider/Syrovatka (2017) zugrunde legt, siegt die Stabilitätsunion klar über die Fiskalunion.

Die hanseatic league hatte an diesem Sieg durchaus ihren Anteil. Auch wenn sie in manchen Stellen von der deutschen Position abweicht, bleibt sie in Fragen der $\mathrm{Zu}$ kunft der Währungsunion letztlich ein fiskalischer Hardliner (The Economist 2018) und damit ein Verbündeter Deutschlands. Kanzlerin Merkel als Regierungschefin der größten Ökonomie der Eurozone konnte Macrons Vorstöße nicht unbeantwortet lassen. Sie musste auf seine Vorschläge reagieren und Zugeständnisse machen, um den deutsch-französischen Motor nicht völlig abzuwürgen - ganz im Gegensatz zur Hansa, die im Schatten Deutschlands dessen Ablehnung einer Fiskalunion offener vortragen konnte. Die Hansa war in der Lage, die auch von Deutschland geteilten Bedenken bezüglich der französischen Vorschläge noch deutlicher geltend zu machen. Deutschland dürfte sich in der Position des Mittlers gefallen - denn für die radikaleren Positionen ist nun die Hansa da. Der Bundesregierung kommt das Emporkommen der Hansa also durchaus zupass.

Zugleich sollte man den Einfluss der Hansa auch nicht überschätzen. Letztlich machen ihre Einwohner/innen gerade einmal 10 Prozent der Bevölkerung der EU aus und sie vertritt weniger als ein Drittel der Mitgliedstaaten. Bei qualifizierter Mehrheitsentscheidung hätte sie demzufolge nicht ausreichend Vetokapazitäten, um Reformen zu blockieren (The Economist 2018). Auch handelt es sich bei der Hansa bisher lediglich um eine informelle Gruppierung, die sich recht lose im Vorfeld von Eurogipfeln meistens zum Abendessen trifft und gemeinsame Positionen abspricht - zumindest wenn man dem offiziellen Twitteraccount (@ HansaInTheEU) der Hansa Glauben schenken mag. Dort findet sich zudem allerlei Skurriles: Die Einigung zwischen Währungskommissar Moscovici und der italienischen Regierung, die nach längerem Streit mit der EU-Kommission doch noch ihr Budgetdefizit nach unten korrigierte, kommentierte die Hansa süffisant mit einem „Weihnachtszeit kommt dieses Jahr früh" (Übers.: S.G.).

Es wird sich zukünftig erweisen müssen, ob die hanseatischen Gemeinsamkeiten auf Fragen der Architektur der Währungsunion begrenzt sind, oder ob sie sich auch auf andere Politikfelder erstrecken werden. Für all jene, die Hoffnungen in 
die Reformierbarkeit der EU von links hegen, dürfte die Formierung der Hansa allerdings einen weiteren Dämpfer bedeuten. Solidarische Umverteilung finanzieller Ressourcen und eine fiskalische Risikoteilung umfangreicher Art wird mit ihr nicht zu machen sein. Damit kann die deutsche Position in der Reformdiskussion weiter Boden gut machen. Der Weg zu einer Fiskalunion bleibt bis auf Weiteres versperrt.

\section{Literatur}

Becker, Joachim (2018): Die Visegrád-4 und die EU.Zwischen Abhängigkeit und partieller Dissidenz. In: PROKLA 48(3): 417-436. DOI: $10.32387 /$ prokla.v48i192.906.

Bieling, Hans-Jürgen (2011): Vom Krisenmanagement zur neuen Konsolidierungsagenda derEU.In: PROKLA 41(2): 173-194. DOI: 10.32387/prokla.v41i163.348.

-/Guntrum, Simon (Hg.) (2019) [im Erscheinen]: Neue Segel, alter Kurs? Die Eurokrise und ibre Folgen für das europäische Wirtschaftsregieren.

BMF (2018): Deutsch-französischer Fahrplan für das Euro-Währungsgebiet. URL: https://www.bundesfinanzministerium. de/Content/DE/Standardartikel/Themen/Europa/2018-06-20-Meseberg-Anl1. pdf?__blob $=$ publicationFile $\& v=2$.

Dullien, Sebastian (2018): Wie weiter mit der Euro-Zone? Aktuelle Reformvorschläge im Vergleich. In: WISO-Diskurs, 20/2018. Bonn.

Dutch Ministry of Finance (2018): ESM Reform. Shared views of the Finance Ministers from the Czech Republic, Denmark, Estonia, Finland, Ireland, Latvia, Lithuania, the Netherlands, Sweden and Slovakia. URL: https://www.rijksoverheid.nl/documenten/kamerstukken/2018/11/02/hanseaticstatement-on-the-esm. Zugriff: 7.1.2018.

ESM (2018): Explainer on ESM reform. URL: https://www.esm.europa.eu/explaineresm-reform.

Europäischer Rat (2018): Euro-Gipfel, 14.12.2018. Wichtigste Ergebnisse. URL: https://www.consilium.europa.eu/de/ meetings/euro-summit/2018/12/14/. Zugriff: 7.1.2019.

F.A.S (2018): „Europa muss handlungsfäbig sein - nach außen und innen". Kanzlerin Merkel im Gespräch. URL: https://www. faz.net/aktuell/politik/inland/kanzlerinangela-merkel-f-a-s-interview-europa-musshandlungsfaehig-sein-15619721.html?pre mium $=0 \times 39$ ea 2 da 7662 fc55ae20248b837 $0 \mathrm{cb} 6 \mathrm{fb}$.

Financial Times (2018a): France ambushes the EU's New Hanseatic League. URL: https://www.ft.com/content/12199bb8f11d-11e8-ae55-df4bf40f9d0d. Zugriff: 8.1.2019.

- (2018b): France warns Hanseatic alliance risksweakening Europe. URL: https://www. ft.com/content/d47c60cc-ef20-11e8-81809cf212677a57.

- (2018c): Hawkish European capitals lobby to beefup eurozone bailout fund. URL: https:// www.ft.com/content/2975alca-ddc3-11e89f04-38d397e6661c. Zugriff: 8.1.2019.

- (2018d): The EU's new Hanseatic League picks its next Brussels battle. URL: https:// www.ft.com/content/ca9dc2dc-c52a-11e8bc21-54264d1c4647. Zugriff: 8.1.2019.

Finnish Ministry of Finance (2018): Finance ministers from Denmark, Estonia, Finland, Ireland, Latvia, Lithuania, the Netherlands and Sweden underline their shared views and values in the discussion on the architecture of the EMU.

Handelsblatt (2018a): „Beharren auf dem Stabilitätspakt ist keine Obsession" - Italien besorgt niederländischen Finanzminister. Wopke Hoekstra im Interview. URL: https://www.handelsblatt.com/politik/ international/wopke-hoekstra-im-interview-beharren-auf-dem-stabilitaetspaktist-keine-obsession-italien-besorgt-niederlaendischen-finanzminister/23686206. html? ticket $=$ ST-1288092-2 $\mathrm{wKusyl540Q-}$ CyrleyVJH-ap4.

- (2018b): Das bedeutet der Reformplan der Eurozone. URL: https://www.handelsblatt. $\mathrm{com} / \mathrm{politik} /$ international/analyse-dasbedeutet-der-reformplan-der-euro -zone/23713898.html?ticket $=$ ST- 414545 
-ylXVTFDP4ILzr6yAkfVn-ap3. Zugriff: 18.12.2018.

Heine, Frederic/Sablowski, Thomas (2015): Zerfällt die Europäische Währungsunion? Handels- und Kapitalverflechtungen, Krisenursachen und Entwicklungsperspektiven der Eurozone. In: Prokla 45(4): 563-592. DOI: $10.32387 /$ prokla.v45i181.201.

Helleiner, Eric (1994): States and the reemergence of global finance. From Bretton Woods to the 1990s. Ithaca NY u.a.

Howarth, David/Quaglia, Lucia (2017): The difficult construction of a European Deposit Insurance Scheme. A step too far in Banking Union? In: Journal of Economic Policy Reform 21(3): 1-20. DOI: $10.1080 / 17487870.2017 .1402682$.

Irish Department of Finance (2018): Capital Markets Union - Shared views of the Finance Ministers from Denmark, Estonia, Finland, Ireland, Latvia, Lithuania, Sweden, and The Netherlands. URL: https://www.finance.gov.ie/wp-content/ uploads/2018/07/180718-Capital-Markets-Union-Paper.pdf. Zugriff: 8.1.2018.

Macchiarelli, Corrado (2017): European Monetary Integration and the EU-UK Relationship. In: Campos, Nauro F./Coricelli, Fabrizio (Hg.): The Economics of UK-EU
Relations. From the Treaty of Rome to the Vote for Brexit. Cham: 79-118.

Macron, Emmanuel (2017): Rede von Staatspräsident Macron an der Sorbonne. Initiative für Europa. Paris, 26.9.2017. URL: https:// de.ambafrance.org/Initiative-fur-EuropaDie-Rede-von-Staatsprasident-Macron-imWortlaut. Zugriff: 4.1.2019.

Quaglia, Lucia/Howarth, David/Liebe, Moritz (2016): The Political Economy of European Capital Markets Union. In:JCMS: Journal of Common Market Studies 54(3): 185-203. DOI: $10.1111 /$ jcms.12429.

Schneider, Etienne/Syrovatka, Felix (2017): Die Zukunft der europäischen Wirtschaftsintegration. Blockierte Vertiefung und wachsende Asymmetrie zwischen Frankreich und Deutschland. In: Prokla 47(4): 653-673. DOI: $10.32387 /$ prokla.v47i189.62.

The Economist (2018): Northern member states unite on eurozone reform. URL: https:// www.economist.com/europe/2018/12/08/ northern-member-states-unite-on-eurozone-reform. Zugriff: 8.1.2019.

Vestergaard, Jakob (2018): Euro Reforms in Deep Coma. URL: https://voxeu.org/debates/commentaries/euro-reforms-deepcoma. Zugriff: 15.1.2019. 